\title{
The clinical utility of joined detection of cancer ratio, cancer ratio plus, Interferon gamma (IFN- $\Upsilon$ ) \& Carcinoembryonic antigen (CEA) in differentiating lymphocytic pleural effusions
}

\author{
Dalia E. ElSharawy ${ }^{1 *}$, Maha M. Hagras² and Rasha A. Khedr ${ }^{3}$
}

\begin{abstract}
Background: The differentiation between malignant (MPE) and tuberculous (TPE) pleural effusions should be considered in any patient with an exudative lymphocytic pleural effusion. A rapid precise diagnosis is valuable as the treatment and prognosis are totally different. The histopathological proof may shorten the time to differential diagnosis. But it may be invasive and costly. The aim of this study is to validate the clinical reliability of joined detection of cancer ratio (serum LDH to pleural ADA), cancer ratio plus (cancer ratio to percentage of pleural fluid lymphocytic count), pleural interferon gamma (pIFN- $\Upsilon$ ), and pleural carcinoembryonic antigen (pCEA) values to differentiate between lymphocytic pleural effusions.

Results: Seventy-eight patients were included with mean age \pm SD $53.09 \pm 9.56$ years old, 49 males and 29 females, diagnosed as $47 \mathrm{MPE}, 24 \mathrm{TPE}$, and 7 others. Cancer ratio at cutoff value of $\geq 22$ and cancer ration plus at cutoff value of $\geq$ 41 can discriminate MPE from any other cause with sensitivity (91.5\%, 93.6\%), specificity (87.5\%, 91.7\%), and diagnostic accuracy $(90.1 \%, 92.9 \%)$ respectively. When the levels of pCEA and pIFN- $\Upsilon$ were combined with cutoff value of cancer ratio, there were powerful diagnostic differentiating results.

Conclusions: Cancer ratio and cancer ratio plus offered valid, efficient, non-invasive, and easy measuring diagnostic tools. On diagnostic uncertainty, the add-on of PCEA in cases of suspected MPE, and pIFN- $Y$ in cases of suspected TPE has a trustable diagnostic efficacy with no need for further investigations.
\end{abstract}

Keywords: Tuberculous pleural effusions, Malignant pleural effusions, Cancer ratio, Carcinoembryonic antigen, Interferon gamma

\section{Background}

The differentiation between malignant and tuberculous pleuritis with effusion should be considered in any patient with an exudative pleural effusion, especially if predominantly lymphocytic [1]. A rapid precise diagnosis is

\footnotetext{
* Correspondence: dalia-elsharawy@hotmail.com

'Department of Chest Diseases, Faculty of Medicine, Tanta University, Tanta, Egypt

Full list of author information is available at the end of the article
}

so valuable as the treatment and prognosis for these two entities are obviously different [2].

Tuberculous pleural effusions (TPE) are primarily an immunologic process with a small number of tuberculous bacilli, so traditional methods of diagnosing tuberculosis fail to recognize a significant proportion besides the late microbiological results of cultures [3]. Although pleural biopsy culture and histology increases diagnostic yield in up to $90 \%$ of tuberculous pleuritis, but its invasiveness limits its use [4]. 
Low diagnostic yield of pleural fluid cytology $(\sim 60 \%)$ and the invasiveness of closed or thoracoscopic pleural biopsy present a challenge in diagnosing malignant pleural effusion (MPE) and any chance for management and improvement [5]. In recent years, advanced researches have been studied to put a precise diagnosis for malignant effusions as tumor markers CEA, CA15-3, CA125, and Cyfra 21-1 in pleural fluid and protein microarray technologies and to differentiate malignant from tuberculous one. But unfortunately, these tests are not widely practiced due to its high cost and lack of availability [6].

Among the routinely performed biochemical tests for investigating exudative pleural effusion; there are serum lactate dehydrogenase (LDH), pleural adenosine deaminase (ADA), and pleural lymphocyte count. This reciprocal change presents an opportunity to combine these results developing a ratio with the diagnostic power to differentiate MPE from TPE in a cost-effective, timely, and universally applicable manner [7].

Adenosine deaminase activity and interferon gamma (IFN-Y) concentrations have been both considered as valuable markers for diagnosing tuberculous pleurisy [8].

Carcinoembryonic antigen (CEA) is one of the most common tumor markers; its level rises in response to tumor existence, development, or even metastasis [9], and this level could be much higher in pleural effusion than that of serum [10].

\section{Aim}

The aim of this study was to validate the clinical reliability of joined detection of cancer ratio (serum LDH to pleural ADA ratio), cancer ratio plus (cancer ratio to pleural fluid lymphocyte count), pleural interferon gamma (pIFN- $\Upsilon$ ) concentration, and pleural carcinoembryonic antigen (pCEA) concentration to differentiate between lymphocytic pleural effusions.

\section{Methods}

\section{Data collection}

This prospective cohort study included all patients admitted to the chest department, Tanta University Hospitals, during the period from August 2018 to July 2019 for management of exudative lymphocytic pleural effusion (based on Light's criteria [11] and pleural lymphocytic count $\geq 50 \%$ of the total white cell count [2]). At least $50 \mathrm{ml}$ of pleural fluid was aspirated within the first $24 \mathrm{~h}$ of admission, sent to clinical pathology laboratory within $2 \mathrm{~h}$ of aspiration, and underwent analysis that included demographic data of patients (age, gender), chemical analysis of pleural fluid $(\mathrm{pH}$, pleural protein, serum protein, pleural glucose level, pleural LDH, serum LDH, pleural fluid differential white cell count, pleural fluid ADA, pleural fluid IFN- $\Upsilon$, and pleural CEA values), microbiological analysis (Ziehl-Neelsen stain of pleural fluid), pleural fluid cytology, and histopathological examination of pleural biopsy if needed. All patients performed chest X-ray immediately after the pleural fluid aspiration to detect any complications. All patients who participated signed an informed written consent form, and the study protocol was approved by the research ethics committee of the Faculty of Medicine, Tanta University.

Pleural effusions were considered tuberculous if:

1) Positive smear for acid-fast bacilli (AFB) of pleural fluid or sputum, or

2) Positive gene expert result of pleural fluid or sputum, or

3) Parietal pleura showed caseous granuloma either by ultrasound-guided core needle pleural biopsy or thoracoscopic pleural biopsy, or

4) High ADA level (> $40 \mathrm{U} / \mathrm{L})$ in the pleural fluid with a positive tuberculin skin test and the effusion responded to treatment with anti-tuberculous drugs (regarding that any other potential causes of exudative lymphocytic effusion were excluded).

Pleural effusions were considered malignant if:

1) Positive cytology of pleural fluid cytology, or

2) Positive histopathological result for malignant cells in the pleural biopsy sample.

Analyzed ratios:

1- The ratio of serum LDH to pleural ADA (cancer ratio);

2- The ratio of cancer ratio to percentage of pleural fluid lymphocytic count (cancer ratio plus);

3- The values of pleural interferon gamma (pIFN-Y) and pleural carcinoembryonic antigen (pCEA) in lymphocytic pleural fluid and their combined detection with cutoff of cancer ratio for defining accurate diagnosis.

\section{Specimen processing}

The biochemical measurements were performed on a clinical chemistry analyzer (Hitachi 917, Boehringer Mannheim, Roche diagnostics, USA) using standard methodology. Cell counting was performed in a Thoma's chamber. Serum LDH was done on AU-480 analyzer using commercial kit (Beckman instrument, Fulteron, USA)

\section{Pleural ADA activity}

ADA activity was detected using a commercial kit (Adenosine Deaminase Assay Kit, San Diego, USA) at $37^{\circ} \mathrm{C}$ 
based on the Berthelot reaction; indophenol complex formed from ammonia liberated from adenosine gave a definitive color that was quantified spectrophotometrically.

\section{Pleural IFN- $Y$ levels}

IFN- $\Upsilon$ levels were measured using immunoassay kit (Becton Dickinson, San Diego, USA); the idea depends on the quantitative enzyme immunoassay technique, which was the dyed microparticles with a maximal emission wavelength of $\sim 650 \mathrm{~nm}$; fluorescence- 3 were coated with antibodies specific to human IFN- $\Upsilon$. IFN- $\Upsilon$ was detected directly using the antibody fused with phycoerythrin, which emits fluorescence at $585 \mathrm{~nm}$ (FL2) by flow cytometry [four-color FACSCalibur flow cytometer (Becton Dickinson Immunocytometry Systems, San Jose, CA, USA)]. The intensity of FL-2 was proportional to the cytokine concentration in the sample, and the results were derived from a calibration curve.

\section{Pleural CEA}

pCEA was determined using the chemiluminescence method on a DXI800 luminescence analyzer (Beckman, Brea, CA, USA) which depends on the phenomenon; when the vibronically excited product of an exoergic reaction relaxes to its ground state with the emission of photon, the intensity is directly proportional to the analyte of interest. The intensity of light is measured in terms of relative light units (RLU).

\section{Statistical analysis}

Data were analyzed using Statistical Program for Social Science (version 20.0, SPSS Inc., Chicago, USA). Quantitative data were expressed as mean \pm standard deviation (SD). Qualitative data were expressed as frequency and percentage. A one-way analysis of variance (ANOVA) was used for comparing between more than two means. Post hoc test was used for multiple comparisons between different variables. Chi square $\left(\chi^{2}\right)$ test of significance compared proportions between two qualitative parameters. Differences were considered statistically significant when $P$ value $<0.05$.

Receiver operating characteristic (ROC curve) analysis was used to find out the overall predictivity of parameter and the best cutoff value with detection of sensitivity and specificity at this cutoff value.

Sensitivity, specificity, positive predictive value (PPV), negative predictive value (NPV), and diagnostic accuracy rate were calculated from the standard definitions.

\section{Results}

Seventy-eight patients with exudative lymphocytic pleural effusion were included with mean age \pm SD $53.09 \pm 9.56$ years old (range 29-72 years old) and male to female percent ratio $62.8 \%: 37.2 \%$ (males no., 49; females no., 29). They were diagnosed as 47 MPE, 24 TPE, and 7 others ( 2 cases of interstitial lung diseases (usual intersistial pneumonitis), 1 case secondary to systemic lupus erythrematosus, 1 case of chronic reumatoid pleurisy, 2 cases with pulmonary embolism, and 1 case with sarcoidosis) based on clinical, radiological, laboratory and/or histopathological proof. MPE were analyzed into primary lung cancer (no., 31 cases), mesothelioma (no., 3), breast cancer (no., 2), lymphoma (no., 4), uterine cancer (no., 2), hepatocellular carcinoma (no., 2), ovarian cancer (no., 2), and cancer colon (no., 1).

The level of serum LDH was significantly higher in MPE than in TPE and other groups while the pleural ADA level and the pleural differential lymphocytic count were higher in TPE than in MPE and other groups (Table 1).

Cancer ratio (S. LDH/ pl. ADA) and cancer ration plus (cancer ratio/ pl. fluid lymphocytic count) were significantly higher in MPE group (mean \pm SD: $52.43 \pm 19.36$ ), $(80.69 \pm 35.88)$ than their levels in "others" group (32.89 \pm $7.52)$, (49.34 \pm 12.71$)$ witch were also higher than their

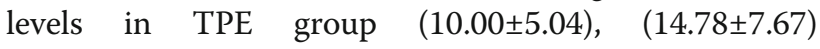
respectively.

The ROC curve analyzed that the cutoff value for cancer ratio to discriminate MPE than other lymphocytic pleural effusions was $\geq 22$ with sensitivity $91.5 \%$, specificity $87.5 \%$, PPV $93.4 \%$, NPV $84 \%$, and accuracy $90.1 \%$. Area under the curve (AUC) was 0.947 . The cutoff value for cancer ratio plus was $\geq 41$ with sensitivity $93.6 \%$, specificity $91.7 \%$, PPV $87.7 \%$, NPV $88 \%$, and accuracy 92.9\%. Area under the curve (AUC) was 0.975 (Fig. 1).

The level of pleural CEA offered an excellent reliable diagnostic point as it was significantly higher in MPE than any other lymphocytic pleural effusions (Table 1), and when combined with cutoff value of cancer ratio, the sensitivity rises to $97.8 \%$, specificity to $91.7 \%$, PPV to $95.8 \%$, NPV to $95.6 \%$, and diagnostic accuracy to $95.7 \%$.

On the other hand, the level of pleural IFN- $\Upsilon$ added a valuable discriminating point for TPE as it was significantly higher in TPE than any other lymphocytic pleural effusions. (Table 1). Its combined detection with cancer ratio at its cutoff value $(\geq 22)$ recorded sensitivity $100 \%$, specificity $95.7 \%$, PPV 92\%, and NPV 100\%, and the diagnostic accuracy reaches $97.2 \%$.

\section{Discussion}

The predominance of lymphocytes in pleural effusion is in favor of malignant or tuberculous pleuritis. Definitive diagnosis should be clear for initiating work-up. While the prompt diagnostic methods as detection of organism in pleural fluid, culture, or histopathological proof of granulomas in cases of tuberculous effusion or malignant cells in case of malignant effusion may shorten the 
Table 1 Chemical characteristics of pleural fluid

\begin{tabular}{|c|c|c|c|c|c|c|c|}
\hline & & Range & Mean \pm SD & $F$ test & $p$ value & & \\
\hline \multirow[t]{3}{*}{$\overline{\text { S. LDH }}$} & Malignant & $415-1390$ & $1046.91 \pm 225.03$ & 39.795 & $0.001^{*}$ & P1 & $0.001^{*}$ \\
\hline & TB & $410-870$ & $643.33 \pm 111.73$ & & & P2 & $0.001^{*}$ \\
\hline & Others & $560-830$ & $707.14 \pm 108.28$ & & & P3 & 0.435 \\
\hline \multirow[t]{3}{*}{ PI. ADA } & Malignant & $11-42$ & $21.87 \pm 6.39$ & 138.064 & $0.001^{*}$ & P1 & $0.001^{*}$ \\
\hline & TB & $35-107$ & $71.88 \pm 19.95$ & & & P2 & 0.911 \\
\hline & Others & $15-33$ & $22.43 \pm 6.00$ & & & P3 & $0.001^{*}$ \\
\hline \multirow[t]{3}{*}{ Pleural fluid Lymphocytic count } & Malignant & $50-100$ & $67.47 \pm 13.10$ & 0.156 & 0.856 & P1 & 0.581 \\
\hline & TB & $50-90$ & $69.21 \pm 12.11$ & & & P2 & 0.961 \\
\hline & Others & $57-85$ & $67.71 \pm 8.96$ & & & P3 & 0.782 \\
\hline \multirow[t]{3}{*}{ Cancer ratio } & Malignant & $16.6-97.5$ & $52.43 \pm 19.36$ & 59.405 & $0.001^{*}$ & P1 & $0.001^{*}$ \\
\hline & TB & $5.4-23.7$ & $10.00 \pm 5.04$ & & & P2 & $0.003^{*}$ \\
\hline & Others & $21.8-44.7$ & $32.89 \pm 7.52$ & & & P3 & $0.001^{*}$ \\
\hline \multirow[t]{3}{*}{ Cancer ratio plus } & Malignant & 21.7-195 & $80.69 \pm 35.88$ & 42.355 & $0.001^{*}$ & P1 & $0.001^{*}$ \\
\hline & TB & $6.8-43.1$ & $14.78 \pm 7.67$ & & & P2 & $0.009^{*}$ \\
\hline & Others & $30.7-64.7$ & $49.34 \pm 12.71$ & & & P3 & $0.006^{*}$ \\
\hline \multirow[t]{3}{*}{ PI. CEA } & Malignant & $86-780$ & $246.40 \pm 147.66$ & 37.926 & $0.001^{*}$ & P1 & $0.001^{*}$ \\
\hline & TB & $2-31$ & $12.71 \pm 8.36$ & & & P2 & $0.001^{*}$ \\
\hline & Others & $2-35$ & $14.43 \pm 11.52$ & & & P3 & 0.972 \\
\hline \multirow[t]{3}{*}{ PI. IFN- $\Upsilon$} & Malignant & $3.5-13.7$ & $7.18 \pm 2.74$ & 68.072 & $0.001^{*}$ & $\mathrm{P} 1$ & $0.001^{*}$ \\
\hline & TB & $132.5-1322.6$ & $542.37 \pm 337.67$ & & & $\mathrm{P} 2$ & 0.991 \\
\hline & Others & $3.8-9.1$ & $6.34 \pm 2.08$ & & & P3 & $0.001^{*}$ \\
\hline
\end{tabular}

*Significant, $P<0.05$

S. $L D H$ serum lactate dehydrogenase enzyme, PI. ADA pleural adenosine deaminase, PI. CEA pleural carcinoembryonic antigen, PI. IFN- $\gamma$ pleural interferon gamma

\section{ROC Curve}

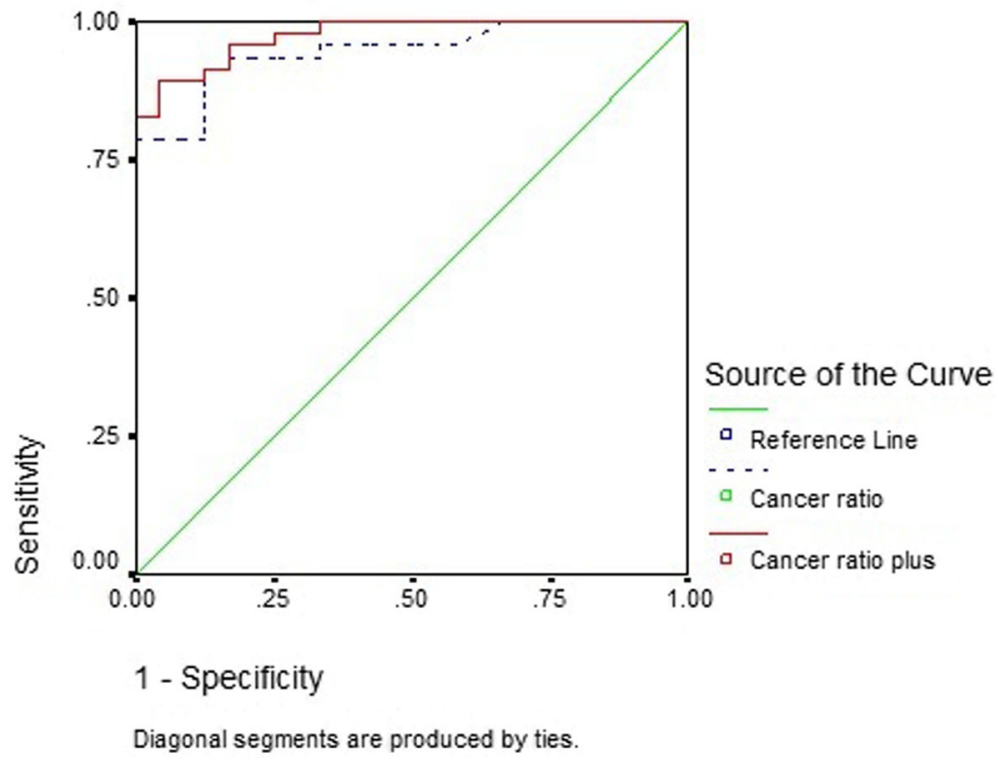

Fig. 1 The ROC curves of cancer ratio and cancer ratio plus for discriminating MPE than any other lymphocytic pleural effusions 
time to differential diagnosis. But these techniques may be invasive or costly as regards required equipment and trained personnel [12].

Lactate dehydrogenase enzyme (LDH) is widely distributed in various types of cells; it performs catalyzation process for conversion of lactate to pyruvic acid for ATP production. When the cells are invaded and destroyed by tumor, LDH is released into the blood, causing increase in plasma LDH level that is expected to occur due to the preferential use of glycolysis for energy, instead of oxidative phosphorylation by tumor cells, which is mediated by LDH. It acts a diagnostic marker in some types of cancer and was associated with survival of MPE [13].

ADA activity is mainly produced by monocytes [14]; its high level in pleural effusion plays an important role in the early diagnosis of tuberculous pleurisy and its differentiation from malignant one [15].

The present study documented the validity of cancer ratio (serum $\mathrm{LDH}$ /pleural $\mathrm{ADA}$ ) as a noninvasive tool in differentiating malignant from any other lymphocytic pleural effusions as the results reported that its cutoff value $\geq 22$ offered sensitivity $91.5 \%$, specificity $87.5 \%$, PPV 93.4\%, NPV 84\%, and diagnostic accuracy $90.1 \%$. AUC was 0.947. These were in accordance with Verma et al. [7] who reported the ROC-derived cutoff level of > 20 for cancer ratio yielded sensitivity, specificity, positive likelihood ratio (PLR), and negative likelihood ratio (NLR) of $0.95,0.85,16,0.13$ respectively [7].

The predominance of small lymphocytes in a pleural effusion indicates that the diagnosis is most likely malignant or tuberculous pleuritis [3]. Previous researches have reported that $>90 \%$ of tuberculous effusions are lymphocytic (lymphocyte count $>50 \%$ of the differential leukocytic count in the pleural fluid) [16]. This study reported that there was no significant difference between TPE and MPE in the mean differential lymphocytic count $(69.21 \pm 12.11,67.47 \pm 13.10$ respectively).

The accuracy of cancer ratio was enhanced when combined with pleural lymphocyte count (cancer ratio: pleural fluid lymphocyte count), what is called cancer ratio plus; its cutoff level in differentiating MPE from other lymphocytic effusions $(\geq 41)$ raised the sensitivity and specificity to $93.6 \%$ and $91.7 \%$ respectively that was in high agreement with Verma et al. [7] who reported that the cutoff level of "cancer ratio plus" of $>30$ had PLR of 41 suggesting that patients with cancer have about 41-fold higher probability of having "cancer ratio plus" of $>30$ in comparison with patients without cancer. In contrast, NLR at this cutoff was found to be 0.06 suggesting that if the "cancer ratio plus" is $<30$, the chance that the diagnosis is cancer is $6 \%$, which is low enough to make the patient unlikely to be diagnosed as having cancer.
Numerous researches have studied the diagnosis of pleural malignancy by rising levels of tumor markers in the pleural effusion such as CEA, CA15-3, CA125, and Cyfra 21-1 [17]. Yet, these techniques are not routinely applied because of high cost, lack of their availability in most centers, and their reported sensitivity and specificity were 0.65 and $0.97,0.57$ and $0.90,0.68$ and 0.83 , and 0.53 and 0.79 , respectively [18]. Our results found that the level of pCEA in MPE was significantly higher in MPE than in any other causes of lymphocytic pleural effusions. When these results were conjugated with the cutoff value of cancer ratio, we can afford excellent diagnostic non-invasive tool of malignant $\mathrm{PE}$ as the sensitivity and specificity reached $97.8 \%$ and $91.7 \%$ respectively, which is higher than each of them separately.

IFN- $\Upsilon$, secreted by antigen-triggered CD4+ lymphocytes, is a key lymphokine that activates macrophages, increasing their bactericidal activity against Mycobacterium tuberculosis [19]. Its level in TPE was significantly higher than any other lymphocytic effusions; that was in accordance with Liu et al. [12] who documented that IFN- $\Upsilon$ offered a more accurate marker than ADA for diagnosis of tuberculous pleurisy, with a higher sensitivity and specificity. However, they added that we cannot depend neither on ADA nor on IFN- $\Upsilon$ solely in the final diagnosis of TPE as they cannot replace bacterial culture with its role in susceptibility testing to guide the optimal antituberculous drugs [12]. When the cutoff value of cancer ratio was combined with results of IFN- $\Upsilon$, we can alleviate any further diagnostic maneuver as their combination offered sensitivity of $100 \%$ and specificity of $95.7 \%$, and the diagnostic accuracy reached $97.2 \%$.

The strength of our study is that the chemical analysis was done within $2 \mathrm{~h}$ of aspiration, so there was no risk of hemolysis that can falsely elevate the serum LDH level with its direct impact on cancer ratio and cancer ratio plus levels. The study included other causes of lymphocytic effusion; but due to their small numbers, we recommend further studies to be done on a larger scale.

\section{Conclusion}

Cancer ratio and cancer ratio plus offered valid, efficient, non-invasive, and easy to measure diagnostic tools-from conventional biochemical analysis of pleural fluid-in differentiation between causes of exudative lymphocytic pleural effusions. On diagnostic uncertainty, the add-on of pCEA in cases of suspected MPE and PIFN- $\Upsilon$ in cases of suspected TPE has a trustable diagnostic efficacy with no need for further investigations that will be accompanied with waste of time, cost, and risk.

\section{Abbreviations}

TPE: Tuberculous pleural effusions; MPE: Malignant pleural effusion; S.

LDH: Serum lactate dehydrogenase; PI. ADA: Pleural adenosine deaminase; 
pIFN-Y: Pleural interferon gamma; pCEA: Pleural carcinoembryonic antigen; AFB: Acid-fast bacilli; RLU: Relative light units; SD: Standard deviation; ANOVA: A one-way analysis of variance; ROC curve: Receiver operating characteristic analysis; PPV: Positive predictive value; NPV: Negative predictive value; AUC: Area under the curve; PLR: Positive likelihood ratio; NLR: Negative likelihood ratio

\section{Acknowledgements \\ Not applicable.}

\section{Authors' contributions}

DEE was responsible for the selection of cases, aspiration of pleural effusion and statistical analysis. MMH was responsible for the specimens processing and data acquisition. RAK was incorporated with the chest department for work-up of malignant pleural effusion cases and reaching its final pathological diagnosis. All authors shared in formatting the study design, manuscript preparation, editing, and reviewing. The authors read and approved the final manuscript.

\section{Funding}

Not applicable.

\section{Availability of data and materials}

All data generated or analyzed during this study are included in this published article [and its supplementary information files].

\section{Ethics approval and consent to participate}

The study protocol was approved by the research ethics committee of Faculty of Medicine, Tanta University, with reference number (32529). A written consent form was obtained from every patient to participate in the research work after sufficient explanation before initiating the study.

\section{Consent for publication}

All patients who participated signed an informed written consent form to publish the data with respect to the privacy of patients and confidentiality of the data as patients' names were replaced by code numbers to confirm their privacy and the results of the study were used only for scientific purpose.

\section{Competing interests}

The authors declare that they have no competing interests.

\section{Author details}

'Department of Chest Diseases, Faculty of Medicine, Tanta University, Tanta, Egypt. ${ }^{2}$ Department of Clinical Pathology, Faculty of Medicine, Tanta University, Tanta, Egypt. ${ }^{3}$ Department of Clinical Oncology, Faculty of Medicine, Tanta University, Tanta, Egypt.

Received: 12 March 2020 Accepted: 8 April 2020

Published online: 20 May 2020

\section{References}

1. Ferrer J (2001) Tuberculous pleural effusion and tuberculous empyema. Semin Respir Crit Care Med 22:637-646

2. Gupta UA, Chhabra SK (2005) Diagnosing tubercular pleural effusions. Chest 127:1078-1079

3. Valde's L, Alvarez D, San Jose' E, Penela P, Valle J.M, Garcia-Pazos J.M, et al (1998) Tuberculous pleurisy: study of 254 patients. Arch Intern Med 158 2017-2021

4. Kirsch CM, Kroe M, Azzi RL, Jensen WA, Kagawa FT, Wehner JH (1997) The optimal number of pleural biopsy specimens for a diagnosis of tuberculous pleurisy. Chest 112:702-706

5. American Thoracic Society (2000). Management of malignant pleural effusions. American Journal of Respiratory and Critical Care Medicine; 162: 1987-2001

6. Porcel JM, Esquerda A, Martınez-Alonso M, Bielsa S, Salud A (2016) Identifying thoracic malignancies through pleural fluid biomarkers: a predictive multivariate model. Medicine 95:e3044-e3051

7. Verma A, Dagaonkar RS, Marshall D, Abisheganaden J, Light RW (2016) Differentiating malignant from tubercular pleural effusion by cancer ratio plus (cancer ratio: pleural lymphocyte count). Can Respir J
8. Greco S, Girardi E, Masciangelo R, Capoccetta GB, Saltini C (2003) Adenosine deaminase and interferon gamma measurements for the diagnosis of tuberculous pleurisy: a meta-analysis. Int J Tuberc Lung Dis 7:777-786

9. Gu Y, Zhai K, Shi HZ (2016) Clinical value of tumor markers for determining cause of pleural effusion. Chin Med J (Engl) 129:253-258

10. Trapé J, Molina R, Sant F (2004) Clinical evaluation of the simultaneous determination of tumor markers in fluid and serum and their ratio in the differential diagnosis of serous effusions. Tumor Biol. 25:276-281

11. Light RW (2002) Clinical practice, pleural effusion. N. Engl. J. Med. 346:1971-1977

12. Liu Y-CH, Lee S, Sh-J, Chen Y.-Sh, Tu H.-Z, Chen B.-Ch, and Huang T.-Sh (2011). Differential diagnosis of tuberculous and malignant pleurisy using pleural fluid adenosine deaminase and interferon gamma in Taiwan. J Microbiol Immunol Infect; 44: 88-94

13. Kayser G, Kassem A, Sienel W, Schulte-Uentrop L, Mattern D, Aumann K, et al. (2010) Lactate-Dehydrogenase 5 is overexpressed in non-small cell lung cancer and correlates with the expression of the transketolase-like protein 1. Diagn Pathol; 5: 22-31

14. Valde's L, San Jose E, Alvarez D and Valle J.M (1996). Adenosine deaminase (ADA) isoenzyme analysis in pleural effusions: diagnostic role, and relevance to the origin of increased ADA in tuberculous pleurisy. Eur Respir J; 9: 747-751

15. Koşar F, Yurt S, Arpınar YB, Şeker B, Kutbay ÖH, Uzun H (2015) The comparative value of pleural fluid adenosine deaminase and neopterin levels in diagnostic utility of pleural tuberculosis. Tuberk Toraks. 63:243-249

16. Vorster MJ, Allwood BW, Diacon AH, Koegelenberg CFN (2015) Tuberculous pleural effusions: advances and controversies. J Thorac Dis 7:981-991

17. Hackbarth JS, Murata K, Reilly W, Algeciras-Schimnich A (2010) Performance of CEA and CA19-9 in identifying pleural effusions caused by specific malignancies. Clin. Biochem. 43:1051-1055

18. Antonangelo L, Sales RK, Cora AP, Acencio MMP, Teixeira LR, Vargas FS (2015) Pleural fluid tumour markers in malignant pleural effusion with inconclusive cytologic results. Current Oncology 22:e336-e341

19. Ribera E, Espanol T, Martinez-Vazquez JM, Ocana I, Encabo G (1990) Lymphocyte proliferation and gamma-interferon production after "in vivo" stimulation with PPD. Differences between tuberculosis and nontuberculosis pleurisy in patients with positive tuberculin skin tests. Chest 97: $1381-1385$

\section{Publisher's Note}

Springer Nature remains neutral with regard to jurisdictional claims in published maps and institutional affiliations.

\section{Submit your manuscript to a SpringerOpen ${ }^{\circ}$ journal and benefit from:}

- Convenient online submission

- Rigorous peer review

- Open access: articles freely available online

- High visibility within the field

- Retaining the copyright to your article

Submit your next manuscript at $\boldsymbol{\nabla}$ springeropen.com 